Claremont Colleges

Scholarship@ Claremont

All HMC Faculty Publications and Research

HMC Faculty Scholarship

$1-1-2009$

\title{
Stability of Traveling Waves in Thin Liquid Films Driven by Gravity and Surfactant
}

Ellen Peterson

North Carolina State University at Raleigh

Michael Shearer

North Carolina State University at Raleigh

Thomas P. Witelski

Duke University

Rachel Levy

Harvey Mudd College

\section{Recommended Citation}

Peterson, E, Shearer, M, Witelski, TP, Levy, R. Stability of traveling waves in thin liquid films driven by gravity and surfactant. In: Tadmor E, Liu JG, Tzavaras A, editors. Hyperbolic Problems: Theory, Numerics and Applications. Proceedings of Symposia in Applied Mathematics. Vol. 67. Pt. 2. Providence: American Mathematical Society, 2009.

This Conference Proceeding is brought to you for free and open access by the HMC Faculty Scholarship at Scholarship @ Claremont. It has been accepted for inclusion in All HMC Faculty Publications and Research by an authorized administrator of Scholarship @ Claremont. For more information, please contact scholarship@cuc.claremont.edu. 


\section{Proceedings of Symposia in Applied Mathematics}

Volume 67, Part 2

\section{Hyperbolic Problems: Theory, Numerics and Applications}

\section{Contributed Talks}

Proceedings of the Twelfth International Conference on Hyperbolic Problems June 9-13, 2008 Center for Scientific Computation and Mathematical Modeling University of Maryland, College Park

Eitan Tadmor Jian-Guo Liu Athanasios Tzavaras Editors 


\title{
EDITORIAL COMMITTEE
}

\author{
Mary Pugh Lenya Ryzhik Eitan Tadmor (Chair) \\ 2000 Mathematics Subject Classification. Primary 35Lxx, 35M10, 35Q30, 35Q60, 35R35, \\ $65 \mathrm{Mxx}, 65 \mathrm{Nxx}, 65 \mathrm{Txx}, 65 \mathrm{Yxx}, 65 \mathrm{Z} 05,74 \mathrm{~B} 20,74 \mathrm{Jxx}_{\mathrm{xx}}, 76 \mathrm{Rxx}, 76 \mathrm{Txx}, 80 \mathrm{~A} 32,80 \mathrm{Mxx}$,
} $85 \mathrm{~F} 05$.

\section{Library of Congress Cataloging-in-Publication Data}

International Conference on Non-linear Hyperbolic Problems (12th : 2008 : University of Maryland)

Hyperbolic problems : theory, numerics, and applications : plenary \& invited talks : Twelfth International Conference on Hyperbolic Problems, June 9-13, 2008, Center for Scientific Computation and Mathematical Modeling, University of Maryland, College Park / Eitan Tadmor, Jian-Guo Liu, Athanasios E. Tzavaras, editors.

p. cm. - (Proceedings of symposia in applied mathematics; v. 67)

Includes bibliographical references and index.

ISBN 978-0-8218-4728-5 (alk. paper)(Set)-ISBN 978-0-8218-4729-9 (alk. paper)(Part 1)ISBN 978-0-8218-4730-5 (alk. paper) (Part 2)

1. Differential equations, Hyperbolic - Congresses. 2. Differential equations, NonlinearCongresses. I. Tadmor, Eitan. II. Liu, Jian-Guo. III. Tzavaras, Athanasios E., 1958IV. Title.

QA377.I563 2008

$515^{\prime} .3535-\mathrm{dc} 22$

2009023286

Copying and reprinting. Material in this book may be reproduced by any means for educational and scientific purposes without fee or permission with the exception of reproduction by services that collect fees for delivery of documents and provided that the customary acknowledgment of the source is given. This consent does not extend to other kinds of copying for general distribution, for advertising or promotional purposes, or for resale. Requests for permission for commercial use of material should be addressed to the Acquisitions Department, American Mathematical Society, 201 Charles Street, Providence, Rhode Island 02904-2294, USA. Requests can also be made by e-mail to reprint-permission@ams.org.

Excluded from these provisions is material in articles for which the author holds copyright. In such cases, requests for permission to use or reprint should be addressed directly to the author(s). (Copyright ownership is indicated in the notice in the lower right-hand corner of the first page of each article.)

(C) 2009 by the American Mathematical Society. All rights reserved.

The American Mathematical Society retains all rights except those granted to the United States Government.

Copyright of individual articles may revert to the public domain 28 years after publication. Contact the AMS for copyright status of individual articles.

Printed in the United States of America.

(6) The paper used in this book is acid-free and falls within the guidelines established to ensure permanence and durability.

Visit the AMS home page at http://www.ams.org/

$10987654321 \quad 141312111009$ 


\title{
Stability of traveling waves in thin liquid films driven by gravity and surfactant
}

\author{
Ellen Peterson, Michael Shearer*, Thomas P. Witelski, and Rachel Levy
}

\begin{abstract}
A thin layer of fluid flowing down a solid planar surface has a free surface height described by a nonlinear PDE derived via the lubrication approximation from the Navier Stokes equations. For thin films, surface tension plays an important role both in providing a significant driving force and in smoothing the free surface. Surfactant molecules on the free surface tend to reduce surface tension, setting up gradients that modify the shape of the free surface. In earlier work $[12,13]$ a traveling wave was found in which the free surface undergoes three sharp transitions, or internal layers, and the surfactant is distributed over a bounded region. This triple-step traveling wave satisfies a system of PDE, a hyperbolic conservation law for the free surface height, and a degenerate parabolic equation describing the surfactant distribution. As such, the traveling wave is overcompressive. An examination of the linearized equations indicates the direction and growth rates of one-dimensional waves generated by small perturbations in various parts of the wave. Numerical simulations of the nonlinear equations offer further evidence of stability to one-dimensional perturbations.
\end{abstract}

\section{Introduction}

The flow of thin liquid films with a free surface of height $h(x, y, t)$ above a flat solid substrate is governed by the thin film equation, which takes the general form

$$
h_{t}+f(h)_{x}=\mu \nabla \cdot(b(h) \nabla h)-\kappa \nabla \cdot(k(h) \nabla \Delta h),
$$

in which $f, b, k$ are non-negative smooth functions vanishing at $h=0$, and $\mu, \kappa$ are dimensionless parameters related to gravity and surface tension, respectively. The flux $f(h)$ includes the effect of driving forces, both body force due to gravity or centrifugal force (important in spin coating [16]), and Marangoni forces, which act on the fluid through surface stress. Marangoni forces can be created in various ways, including the flow of a second fluid above the film [17], or variations in surface tension. The latter can be induced using a temperature gradient $[\mathbf{3}]$, or with a surfactant applied to the surface [7]. Each of these contexts introduces

Research supported by NSF Grants DMS 0604047, DMS 0636590 RTG.

Research supported by NSF Grant DMS 0604047.

Research supported by NSF Grants DMS-0239125 CAREER and DMS-0244498 FRG.

Research supported by NSF Grants DMS-0239125 CAREER and DMS-0244498 FRG.

The authors wish to thank Kevin Zumbrun for helpful discussions and suggestions. 
interesting mathematical issues, including challenges for the theory of hyperbolic PDE (in the limit of vanishing $\kappa$ and $\mu$ ).

For flow on an incline, driven against gravity by a temperature gradient, we find $f(h)=h^{2}-h^{3}$ is non-convex, and $b(h)=k(h)=\frac{1}{3} h^{3}[\mathbf{3}, \mathbf{1 1}]$. The PDE admits traveling waves that are smooth counterparts of undercompressive shocks. Moreover, although there is now a comprehensive theory of scalar conservation laws admitting undercompressive (otherwise known as nonclassical) shocks, using the notion of kinetic relation [8], equation (1.1) does not fit into the theory, and a new theory incorporating a nucleation condition (employed previously only for systems that change type [1]) was devised that explains various anomalies in longtime behavior observed in numerical simulations $[\mathbf{9}, \mathbf{1 1}]$.

We consider thin film flow driven down an incline by gravity and Marangoni stress induced by a layer of surfactant. The surfactant reduces surface tension in proportion to the concentration or density $\Gamma(x, y, t) \geq 0$ of surfactant molecules. Thus, the concentration gradient $\nabla \Gamma$ enters $f(h)$ in equation (1.1). Additionally, we need an equation to model the transport and spreading of the surfactant itself. The lubrication approximation to the Navier-Stokes equations gives the following system of PDE for the height $h(x, y, t)$ of the free surface and surfactant concentration $\Gamma(x, y, t)$, where $x$ measures distance down the incline, and the $y$ axis is in the transverse horizontal direction.

$$
h_{t}+\left(\frac{1}{3} h^{3}\right)_{x}-\nabla \cdot\left(\frac{1}{2} h^{2} \nabla \Gamma\right)=\mu \nabla \cdot\left(\frac{1}{3} h^{3} \nabla h\right)-\kappa \nabla \cdot\left(\frac{1}{3} h^{3} \nabla \Delta h\right)
$$

$$
\Gamma_{t}+\left(\frac{1}{2} h^{2} \Gamma\right)_{x}-\nabla \cdot(h \Gamma \nabla \Gamma)=\mu \nabla \cdot\left(\frac{1}{2} h^{2} \Gamma \nabla h\right)-\kappa \nabla \cdot\left(\frac{1}{2} h^{2} \Gamma \nabla \Delta h\right)+\delta \Delta \Gamma .
$$

The parameter $\mu$ measures the diffusive effect of gravity in pressing the film against the substrate, thereby creating a tendency for the film to spread; $\kappa$ measures the smoothing effect of surface tension. Each of these effects contributes to smoothing the free surface height; their role in equation $(1.2 \mathrm{~b})$ for $\Gamma$ is to modify the transport of surfactant. Smoothing of the surfactant profile is governed by the coefficient $\delta$, which is proportional to the inverse of the Peclet number, modeling the diffusion of surfactant molecules on the surface of the film [18]. Apart from this molecular diffusion term, all the terms in the system represent transport by the fluid motion. The transport speed for $h$ is the depth-averaged component $u$ of the fluid velocity parallel to the incline, whereas the transport speed of the surfactant is the surface speed, the value of $u$ at the free surface.

For much of this paper, we shall be concerned with the unregularized equations, in which $\mu=\kappa=\delta=0$, in one space dimension:

$$
\begin{aligned}
& h_{t}+\left(\frac{1}{3} h^{3}\right)_{x}-\left(\frac{1}{2} h^{2} \Gamma_{x}\right)_{x}=0 \\
& \Gamma_{t}+\left(\frac{1}{2} h^{2} \Gamma\right)_{x}-\left(h \Gamma \Gamma_{x}\right)_{x}=0 .
\end{aligned}
$$

This system is hyperbolic-degenerate parabolic: equation (1.3a) is a scalar conservation law for $h$, while equation (1.3b) is a parabolic equation for the evolution of $\Gamma$ that degenerates at $\Gamma=0$. Since we shall always assume $h>0$ everywhere, we avoid any singularities associated with dry patches $(h=0)$; this also avoids the interesting but difficult scientific issues surrounding motion of a contact line at the leading or trailing edge of a droplet [5]. 
In [12], jump conditions for system (1.3) were formulated, and a surprising new traveling wave was found, in which $h$ is piecewise constant with three jumps, and $\Gamma$ is continuous and piecewise linear, with jumps in $\Gamma_{x}$ corresponding to the jumps in $h$. We call this a triple-step traveling wave. In this paper, we present preliminary results related to triple-step traveling waves, and the smoother versions for which the parameters $\mu, \kappa, \delta$ are not all zero. In $\S 2$, we summarize the existence of the traveling waves and their overcompressive property; in $\S 3$ we analyze the linearization of the PDE system about sections of the wave in which $h$ is constant and $\Gamma$ is linear. In this section, we also give numerical results showing how small perturbations propagate within the wave. In the final section, we discuss the implications of the results, and directions for further research.

\section{Traveling waves}

In this section, we summarize the construction $[\mathbf{1 2}, \mathbf{1 3}]$ of triple-step traveling wave solutions of (1.3), in which $h$ is piecewise constant, and $\Gamma$ is continuous and piecewise linear. Then we discuss smooth traveling waves, for which existence is known for small mass of surfactant or in the absence of capillarity.

Triple-step traveling waves.

In [12], traveling waves were found for the system (1.3). Let $h_{L}, h_{R}$ be given upstream and downstream heights of the film, and let $q_{\text {crit }}=\frac{1}{2}(\sqrt{3}-1)$, a threshold for the ratio $q=h_{R} / h_{L}$. Then, provided $h_{R} / h_{L}<q_{\text {crit }}$, the traveling waves are given by explicit formulae as functions of the traveling wave variable $\eta=x-s t$, with speed

$$
\begin{gathered}
s=\frac{1}{3}\left(h_{L}^{2}+h_{L} h_{R}+h_{R}^{2}\right), \\
h(\eta)=\left\{\begin{array}{ll}
h_{L} & \eta<\eta_{1} \\
h_{1} & \eta_{1}<\eta<0 \\
h_{2} & 0<\eta<\eta_{2} \\
h_{R} & \eta_{2}<\eta
\end{array} \quad \Gamma(\eta)= \begin{cases}0 & \eta \leq \eta_{1} \\
\Gamma_{\max }+G_{1} \eta & \eta_{1} \leq \eta \leq 0 \\
\Gamma_{\max }+G_{2} \eta & 0 \leq \eta \leq \eta_{2} \\
0 & \eta_{2} \leq \eta\end{cases} \right.
\end{gathered}
$$

Here, $\eta_{1}, \eta_{2}$ are related to $\Gamma_{\max }$ so as to make $\Gamma(\eta)$ continuous,

$$
\eta_{1}=-\frac{\Gamma_{\max }}{G_{1}}<0, \quad \eta_{2}=-\frac{\Gamma_{\max }}{G_{2}}>0 .
$$

The formula (2.2) is constrained by suitable jump conditions and the requirement that the individual pieces satisfy the PDE system (1.3). From this, it follows that the intermediate heights $h_{1}>h_{2}>0$ are the two positive values of $h$ satisfying the cubic equation $6 s h-h^{3}=4 h_{L} h_{R}\left(h_{L}+h_{R}\right.$ ), and the intermediate slopes (surfactant concentration gradients) $G_{1}>0>G_{2}$ are specific functions of $h_{L}, h_{R}$, most conveniently expressed in terms of $h_{1}, h_{2}, s$ :

$$
G_{1}=\frac{h_{1}^{2}-2 s}{2 h_{1}}>0, \quad G_{2}=\frac{h_{2}^{2}-2 s}{2 h_{2}}<0 .
$$

The total mass of surfactant in the traveling wave solution is given by

$$
m=\int_{-\infty}^{\infty} \Gamma d \eta=\frac{1}{2} \Gamma_{\max }\left(\eta_{2}-\eta_{1}\right)=\frac{1}{2} \Gamma_{\max }^{2}\left(G_{1}^{-1}-G_{2}^{-1}\right) \geq 0 .
$$


Thus, for given upstream and downstream heights $h_{L}, h_{R}$, there is a one-parameter family of traveling waves; either the mass $m$ or $\Gamma_{\max }$ may be used to parametrize the traveling waves. The structure of the solution is shown in Figure 1.
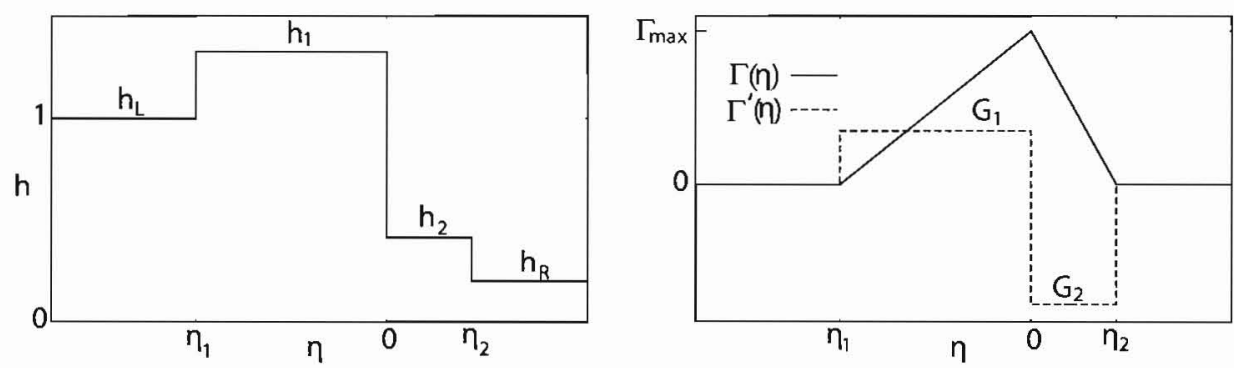

FIGURE 1. Triple-step traveling wave. Height, surfactant concentration and gradient.

The traveling waves are overcompressive in the sense that small perturbations introduced ahead of the wave travel at a slower speed than the wave whereas perturbations behind travel faster. More precisely, linearizing the PDE system about the constant upstream or downstream heights $h=h_{L}$ or $h=h_{R}$ and $\Gamma=0$, we obtain a linear system for the perturbations $\bar{h}, \bar{\Gamma}$ :

$$
\begin{gathered}
\partial_{t} \bar{h}+h^{2} \partial_{x} \bar{h}=\frac{1}{2} h^{2} \partial_{x x} \bar{\Gamma} \\
\partial_{t} \bar{\Gamma}+\frac{1}{2} h^{2} \partial_{x} \bar{\Gamma}=0 .
\end{gathered}
$$

Let $\bar{h}_{0}(x), \bar{\Gamma}_{0}(x)$ be initial data:

$$
\bar{h}(x, 0)=\bar{h}_{0}(x) ; \quad \bar{\Gamma}(x, 0)=\bar{\Gamma}_{0}(x) .
$$

Then the solution of the Cauchy problem (2.6), (2.7) involves traveling waves with constant speeds $c_{1}=\frac{1}{2} h^{2}, c_{2}=h^{2}$ :

$$
\bar{h}(x, t)=\bar{h}_{0}\left(x-c_{2} t\right)+\bar{\Gamma}_{0}^{\prime}\left(x-c_{1} t\right)-\bar{\Gamma}_{0}^{\prime}\left(x-c_{2} t\right) ; \quad \bar{\Gamma}(x, t)=\bar{\Gamma}_{0}\left(x-c_{1} t\right) .
$$

Due to the threshold constraint $h_{R} / h_{L}<q_{\text {crit }}$, we find that the wave speeds $h^{2}, \frac{1}{2} h^{2}$ are greater than the traveling wave speed $s$ for $h=h_{L}$, and $s$ is larger than both wave speeds for $h=h_{R}$.

\section{Smooth traveling waves.}

The non-negative parameters $\mu, \kappa, \delta$ control smoothness of the traveling waves. For $\kappa=0$, it is straightforward to examine a two-dimensional phase plane to show that there is a one-parameter family of traveling waves. Each traveling wave is smooth (as a function of $\eta=x-s t$ ) for $\mu>0, \delta>0$, but has reduced smoothness when one of these parameters is zero. This is explained in [13]. For $\kappa>0$, the situation is more complicated, and there are open issues of existence and uniqueness. A recent paper of Schecter and Manukian [15] provides existence of traveling waves provided a transversality condition is satisfied. Moreover, this condition guarantees that all the traveling waves are parametrized smoothly by (small values of) $m$ or $\Gamma_{\max }$. 


\section{One dimensional stability of triple-step traveling waves}

For a nonlinear conservation law

$$
h_{t}+f(h)_{x}=0,
$$

in which $f(h)$ is nonlinear, traveling waves are single-step shocks joining two constants $h=h_{L}, h=h_{R}$, with speed $s=\left(f\left(h_{L}\right)-f\left(h_{R}\right)\right) /\left(h_{L}-h_{R}\right)$. Stability is established by considering perturbations of the two constant states, solving the initial value problem, together with the Rankine-Hugoniot jump condition. For a scalar equation, this is comparatively straightforward. Let $h=h_{0}$ be a constant, and consider a small perturbation $u: h=h_{0}+u$. Linearizing about $h_{0}$, we find that $u$ satisfies the linear transport equation

$$
u_{t}+f^{\prime}\left(h_{0}\right) u_{x}=0
$$

so that $u(x, t)=u_{1}(x-c t)$, where $c=f^{\prime}\left(h_{0}\right)$ is the characteristic speed. Under the Lax entropy condition

$$
f^{\prime}\left(h_{R}\right)<s<f^{\prime}\left(h_{L}\right)
$$

perturbations to the shock wave, initiated away from the shock itself, approach the shock from both sides, modifying the shock location; the shock is considered stable. Proving nonlinear stability involves showing that the full nonlinear problem is well posed. This theory is well established for scalar equations and for strictly hyperbolic systems of equations [14], in which $u \in \mathbb{R}^{n}$, and $f: \mathbb{R}^{n} \rightarrow \mathbb{R}^{n}$ is continuously differentiable, with distinct and real characteristic speeds, the eigenvalues of the Jacobian $d f(u)$. In our problem, we have a system of two equations for unknowns $h$ and $\Gamma$, but the second equation is degenerate parabolic rather than hyperbolic. Traveling waves for the hyperbolic-parabolic system involve constant $h$ and linear $\Gamma$. The linear system resulting from linearization of (1.3) consequently has nonconstant coefficients, since the underlying solution is not constant in $\Gamma$. The local dispersion relation for this system, obtained by freezing the variable coefficients, gives some information about the short-time evolution of localized perturbations.

3.1. The linearized equations. Consider the triple-step traveling wave (2.2) of the unregularized system (1.3). Between jumps in $h$ and $\Gamma_{x}, h$ is constant and $\Gamma$ is linear. In particular, in those sections in which $\Gamma$ is not identically zero, we have

$$
h=h_{i}, \quad \Gamma=G_{i}(x-s t), \quad s=\frac{1}{2} h_{i}^{2}-h_{i} G_{i}, \quad i=1,2,
$$

(where we have used (2.4) to express the wave speed $s$ in terms of the constants $h_{i}, G_{i}$, and have translated $x$ by $\eta_{i}$ ). For $a>0$ the equations (1.3) are unchanged by the transformation

$$
h \rightarrow a h, \quad \Gamma \rightarrow a^{2} \Gamma, \quad x \rightarrow a x, \quad t \rightarrow t / a .
$$

Consequently, as long as we consider the $i^{\text {th }}$ section in isolation, we can take $h_{i}=1$, so that $s=\frac{1}{2}-G$, in which we write $G=G_{i}$.

Now consider perturbations of a section of the traveling wave

$$
h=1+u, \quad \Gamma=G(x-s t)+v .
$$

To maintain $\Gamma>0$, we consider $x-s t>0$ if $G>0$, and $x-s t<0$ if $G<0$. Substituting (3.3) into the PDE system, and retaining only terms that are linear 
in $u, v$, we obtain the linear system

$$
\begin{aligned}
u_{t}+(1-G) u_{x}-\frac{1}{2} v_{x x} & =0 \\
v_{t}+\left(\frac{1}{2}-G\right) v_{x}-\left[(x-s t) G\left(v_{x}-(1-G) u\right)\right]_{x} & =0 .
\end{aligned}
$$

It is convenient to consider these equations in a frame moving with constant speed $s=\frac{1}{2}-G$. Accordingly, let $\eta=x-\left(\frac{1}{2}-G\right) t$ :

$$
\begin{aligned}
u_{t}+\frac{1}{2} u_{\eta}-\frac{1}{2} v_{\eta \eta} & =0 \\
v_{t}-\left[\eta G\left(v_{\eta}-(1-G) u\right)\right]_{\eta} & =0 .
\end{aligned}
$$

Carrying out the differentiation in $(3.5 \mathrm{~b})$ and then freezing the coefficient $\eta=\gamma$, we obtain the constant coefficiant linear system

$$
\begin{aligned}
u_{t}+\frac{1}{2} u_{\eta}-\frac{1}{2} v_{\eta \eta} & =0 \\
v_{t}-G v_{\eta}+G(1-G) u-\gamma G v_{\eta \eta}+\gamma G(1-G) u_{\eta} & =0 .
\end{aligned}
$$

We seek solutions of the form

$$
u=\tilde{u} e^{i \lambda t+i \xi \eta}, \quad v=\tilde{v} e^{i \lambda t+i \xi \eta}, \quad \lambda=\alpha+i \beta,
$$

where $\xi>0$ is the wave number, or spatial frequency. Writing

$$
e^{i \lambda t+i \xi \eta}=e^{-\beta t} e^{i \xi\left(\frac{\alpha}{\xi} t+\eta\right)}=e^{-\beta t} f(\eta-c t), \quad c=\frac{-\alpha}{\xi},
$$

isolates the wave speed $c$ of the perturbation (relative to $s=\frac{1}{2}-G$ ); $\beta>0$ indicates decay in time and $\beta<0$ growth. Substituting (3.7) into the linear PDE system (3.6), we get simultaneous linear homogeneous equations for $\tilde{u}$ and $\tilde{v}$ which have a solution if and only if the determinant of the coefficient matrix is zero; this leads to the dispersion relation

$$
\begin{aligned}
& \left|\begin{array}{cc}
i\left(\lambda+\frac{1}{2} \xi\right) & \frac{1}{2} \xi^{2} \\
G(1-G)(1+i \xi \gamma) & i(\lambda-G \xi)+\gamma G \xi^{2}
\end{array}\right|= \\
& -\lambda^{2}+\lambda G \xi-\frac{1}{2} \lambda \xi+i \lambda \gamma G \xi^{2}+i \frac{1}{2} \gamma G^{2} \xi^{3}+\frac{1}{2} G^{2} \xi^{2}=0 .
\end{aligned}
$$

Since this is a quadratic equation in $\lambda$, there are two (complex) solutions for each choice of the other parameters. Recalling that $\lambda=\alpha+i \beta$, we get equations for the real and imaginary parts:

$$
\begin{gathered}
-\alpha^{2}+\beta^{2}+\alpha \xi\left(G-\frac{1}{2}\right)-\beta \gamma G \xi^{2}+\frac{1}{2} G^{2} \xi^{2}=0 \\
-2 \alpha \beta+\beta \xi\left(G-\frac{1}{2}\right)+\alpha \gamma G \xi^{2}+\frac{1}{2} \gamma G^{2} \xi^{3}=0 .
\end{gathered}
$$

Completing the square for both of these equations

(3.11a) $\left(\beta-\frac{1}{2} \gamma G \xi^{2}\right)^{2}-\left(\alpha-\frac{1}{2} \xi\left(G-\frac{1}{2}\right)\right)^{2}=-\frac{1}{4} \xi^{2}\left(3 G^{2}-G+\frac{1}{4}\right)+\frac{1}{4} \gamma^{2} G^{2} \xi^{4}$

$$
\left(\alpha-\frac{1}{2} \xi\left(G-\frac{1}{2}\right)\right)\left(\beta-\frac{1}{2} \gamma G \xi^{2}\right)=\frac{1}{2} \gamma G\left(G-\frac{1}{4}\right) \xi^{3} .
$$

These equations describe hyperbolas in the $(\alpha, \beta)$ plane with common center,

$$
\left(\alpha_{0}, \beta_{0}\right)=\left(\frac{1}{2} \xi\left(G-\frac{1}{2}\right), \frac{1}{2} \gamma G \xi^{2}\right) .
$$

Equation (3.11a) represents a hyperbola with asymptotes at $45^{\circ}$; $(3.11 \mathrm{~b})$ is a hyperbola with asymptotes parallel to the coordinate axes. The orientations of these hyperbolas are determined by the sign of the right hand sides of the respective equations, which depend on the following conditions. 
(i) The right hand side of (3.11a) is positive if and only if

$$
\gamma^{2} \xi^{2}>3-\frac{1}{G}+\frac{1}{4 G^{2}}
$$

(ii) The right hand side of $(3.11 \mathrm{~b})$ is positive if and only if

$$
G>\frac{1}{4} \text {. }
$$

We also note from examining (3.10) that without loss of generality we can take $\xi>0$ due to the symmetry of the solution; if $\xi \rightarrow-\xi$ then $\alpha \rightarrow-\alpha$ but the sign of $c=\frac{-\alpha}{\xi}$ remains unchanged.

3.2. Analysis of the dispersion relation (3.11). In analyzing the dispersion relation in the form (3.11), we consider $\gamma$ and $G$ fixed, and determine the behavior of solutions $(\alpha, \beta)$ on the wavenumber $\xi$. We are specifically interested in the signs of $\alpha$ and $\beta$, rather than their magnitude, so we focus on identifying in which quadrant of the $(\alpha, \beta)$ plane the solutions lie. These solutions correspond to the intersection of the hyperbolas. As remarked above, there are two intersections, corresponding to two waves with different speeds and growth/decay rates.

We restrict attention to values of $\gamma, G$ satisfying $\gamma G>0$, with $-\infty<G<0$, or $0<G<\frac{1}{3}$. To explain these inequalities, first note that $\gamma G>0$ is consistent with the two interior levels $h=h_{1}, h=h_{2}$ in the traveling wave. Next observe that, as $h_{R} \rightarrow 0^{+}$in the traveling wave, $h_{1} \rightarrow \sqrt{2} h_{L}, h_{2} \rightarrow 0$ and $G_{1} \rightarrow \frac{\sqrt{2} h_{L}}{3}, G_{2} \rightarrow-\infty$. Thus, with the normalization $h_{1}=1$, we have $G_{1}<\frac{1}{3}$. This upper bound on $G$ also guarantees that when (3.13) is satisfied, then $\gamma^{2} \xi^{2}>2$. Note that the other limit $\frac{h_{R}}{h_{L}} \rightarrow q_{\text {crit }}$ causes $h_{1}, h_{2} \rightarrow h_{L}$ which implies $G_{1}, G_{2} \rightarrow 0$. We deduce that the center $\left(\alpha_{0}, \beta_{0}\right)$ of the two hyperbolas, given by (3.12), lies in the second quadrant.

We first consider the case $0<G<\frac{1}{3}, \gamma>0$ corresponding to the portion $h=h_{1}, \Gamma=G_{1}(x-s t)$ of the traveling wave. It is convenient to define functions $\phi, \psi$ of $G$ :

$$
\phi(G)=\frac{2\left(G-\frac{1}{2}\right)^{2}}{G(1-3 G)}, \quad \psi(G)=3-\frac{1}{G}+\frac{1}{4 G^{2}} .
$$

Proposition 3.1. Suppose $0<G<\frac{1}{3}, \gamma>0$.

- If $\gamma^{2} \xi^{2}>\phi(G)$, then both intersections of the hyperbolas occur in quadrant II. In this case, both waves decay and move to the right (in the frame moving with the traveling wave speed).

- If $\gamma^{2} \xi^{2}<\phi(G)$, then one intersection of the hyperbolas is in quadrant $I$ and one intersection is in quadrant II. Both waves decay but one moves to the right and the other moves to the left.

Proof: We consider various cases based on the structure of the hyperbolas, determined by the sign of the right hand side of (3.11). The cases are illustrated in Figures 2,3. In order to determine in which quadrant the hyperbolas intersect we compare the intersections of the hyperbolas with the coordinate axes. Figures 2 and 3 show examples of where these intersections may occur.

In the figures, dashed lines represent the asymptotes, and $\mathrm{C}$ and $\mathrm{D}$ represent the intersections of the two hyperbolas. The intersections of the hyperbola (3.11a) with the $\alpha$ and $\beta$ axis are labeled $\alpha_{a}^{ \pm}$and $\beta_{a}^{ \pm}$. Intersections of (3.11b) are $\alpha_{b}$ and $\beta_{b}$. 

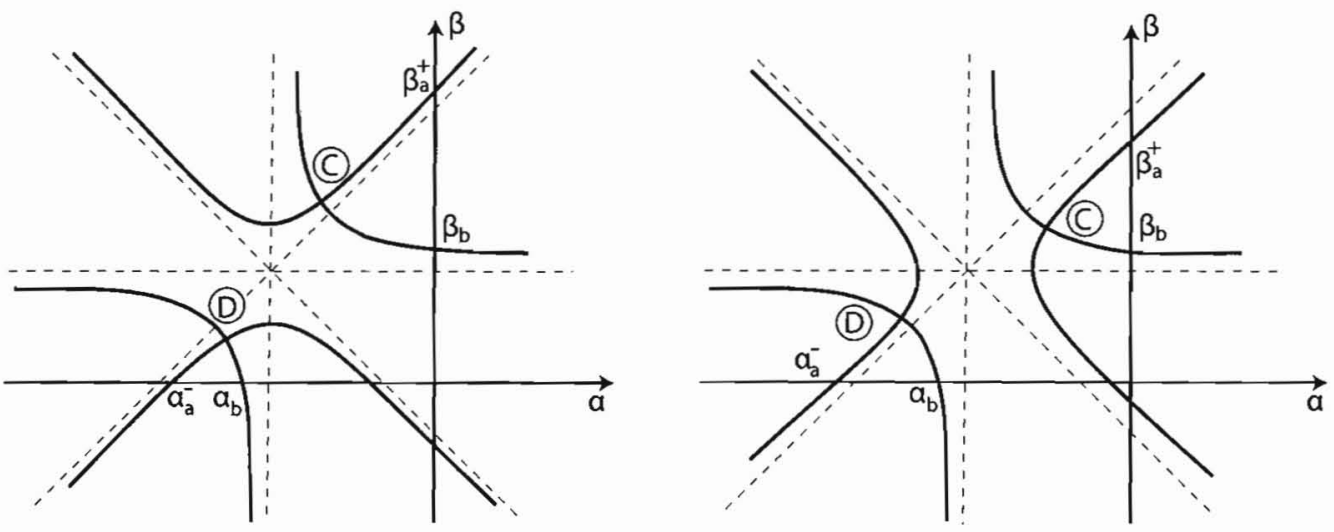

Figure 2. $G>\frac{1}{4}$. Left: $\gamma^{2} \xi^{2}>\psi(G) ;$ Right: $\gamma^{2} \xi^{2}<\psi(G)$.

Setting $\beta=0$ in (3.11a), (3.11b) we find

$$
\alpha_{a}^{ \pm}=\frac{\xi}{2}\left[G-\frac{1}{2} \pm \sqrt{\left(G-\frac{1}{2}\right)^{2}+2 G^{2}}\right], \quad \alpha_{b}=-\frac{1}{2} \xi G .
$$

Similarly, setting $\alpha=0$ in (3.11a), (3.11b) we find

$$
\beta_{a}^{ \pm}=\frac{\xi G}{2}\left(\gamma \xi \pm \sqrt{\gamma^{2} \xi^{2}-2}\right), \quad \beta_{b}=\frac{1}{2} \gamma G \xi^{2}-\frac{\gamma G\left(G-\frac{1}{4}\right) \xi^{2}}{G-\frac{1}{2}} .
$$

Thus

$$
\alpha_{a}^{ \pm}-\alpha_{b}=\frac{\xi}{2}\left[2 G-\frac{1}{2} \pm \sqrt{\left(G-\frac{1}{2}\right)^{2}+2 G^{2}}\right]
$$

(3.18b) and

$$
\beta_{a}^{ \pm}-\beta_{b}=\frac{G \xi\left[ \pm\left(G-\frac{1}{2}\right) \sqrt{\gamma^{2} \xi^{2}-2}+2 \gamma \xi\left(G-\frac{1}{4}\right)\right]}{2\left(G-\frac{1}{2}\right)} .
$$

Now we consider the cases separately, labeled as in Figures 2,3.

Case I: $G>\frac{1}{4}$.

First consider $\gamma^{2} \xi^{2}>\psi(G)$ (see Fig. 2(Left)). Since $G \xi>0$ and $G<\frac{1}{3}$, the sign of $\left(3.18 \mathrm{~b}^{+}\right)$, is determined by the sign of

$$
P(G, \gamma \xi) \equiv\left(G-\frac{1}{2}\right) \sqrt{\gamma^{2} \xi^{2}-2}+2 \gamma \xi\left(G-\frac{1}{4}\right),
$$

Since $P(G, \gamma \xi)=0$ when

$$
\gamma^{2} \xi^{2}=\frac{2\left(G-\frac{1}{2}\right)^{2}}{G(1-3 G)}=\phi(G),
$$

we conclude for the intersection $\mathrm{C}$,

- If $\gamma^{2} \xi^{2}>\phi(G)$ then $\beta_{a}^{+}-\beta_{b}>0$. The intersection is in quadrant II.

- If $\gamma^{2} \xi^{2}<\phi(G)$ then $\beta_{a}^{+}-\beta_{b}<0$. The intersection is in quadrant I.

Note that in the companion case, $G>\frac{1}{4}, \gamma^{2} \xi^{2}<\psi(G)$ (see Fig. 2(Right)), $\beta_{a}^{ \pm}$are complex when $\gamma^{2} \xi^{2}<2$ because then the hyperbola does not intersect the 
$\beta$ axis. The intersection $\mathrm{C}$ is then necessarily in quadrant I. For $\gamma^{2} \xi^{2}>2$, the argument above implies that $\mathrm{C}$ is in quadrant II.

Now we examine the intersection $D$ in Figure 2. This intersection occurs in either quadrant II if $\alpha_{a}^{-}<\alpha_{b}$ or quadrant III if $\alpha_{a}^{-}>\alpha_{b}$. But $\alpha_{a}^{-}-\alpha_{b}$ changes sign when

$$
Q(G) \equiv 2 G-\frac{1}{2}-\sqrt{\left(G-\frac{1}{2}\right)^{2}+2 G^{2}}=0 .
$$

Solving for $G$ we find that $G=0$ or $G=1$. But $Q<0$ when $G=0$ so $Q<0$ for $0<G<1$. Consequently, the intersection D is in quadrant II.

Case II : $0<G<\frac{1}{4}$.
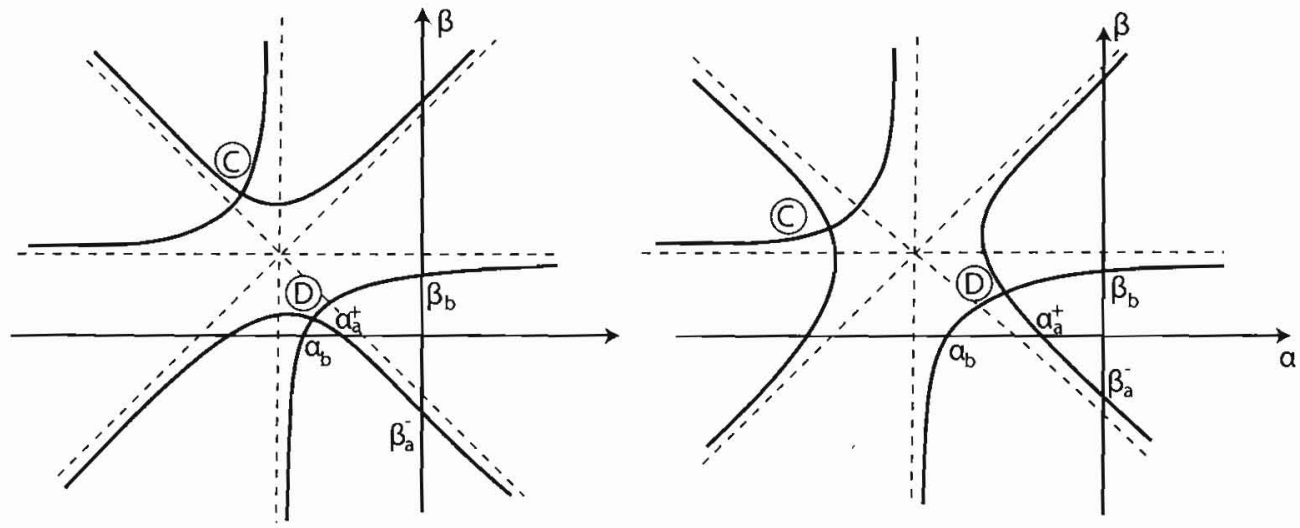

Figure 3. $G<\frac{1}{4}$. Left: $\gamma^{2} \xi^{2}>\psi(G)$; Right: $\gamma^{2} \xi^{2}<\psi(G)$.

From the location of the center (in the second quadrant of Figure 3), and the orientation of the asymptotes, it is clear that intersection $\mathrm{C}$ is in the second quadrant, and we can focus on intersection $D$. In this case, the sign of $\alpha_{a}^{+}-\alpha_{b}$ is determined by the sign of

$$
\tilde{Q}(G)=2 G-\frac{1}{2}+\sqrt{\left(G-\frac{1}{2}\right)^{2}+2 G^{2}},
$$

which has zeros as $G=0$ and $G=1$, but when $G=1, \tilde{Q}>0$. Thus $\tilde{Q}>0$ for $0<G<1$ and $\alpha_{a}^{+}-\alpha_{b}>0$ which means the intersection occurs in quadrant I or II.

From (3.18b), in the case $\gamma^{2} \xi^{2}>\psi(G)$ (see Fig. 3 Left), for which both roots are real, we deduce:

- If $\gamma^{2} \xi^{2}>\phi(G)$ then $\beta_{a}^{-}-\beta_{b}<0$. Thus the intersection D is in quadrant II.

- If $\gamma^{2} \xi^{2}<\phi(G)$ then $\beta_{a}^{-}-\beta_{b}>0$. Thus the intersection $\mathrm{D}$ is in quadrant I.

The only difference in the companion case $0<G<\frac{1}{4}, \gamma^{2} \xi^{2}<\psi(G)$ (see Fig. 3 Right), is that $\beta_{a}^{ \pm}$may be complex, when the hyperbola does not intersect the $\beta$ axis. But then the intersection D clearly is in quadrant 1; otherwise, the intersection $\mathrm{D}$ is in quadrant I or II as above. This completes the proof. 
Next, we consider the case $G<0, \gamma<0$ corresponding to the portion $h=$ $h_{2}, \Gamma=G_{2}(x-s t)$ of the traveling wave. In this case, the right hand side of $(3.11 \mathrm{~b})$ is automatically negative. Thus the only two cases to consider are dependent on the sign of the right hand side of (3.11a). Figure 3 shows the structure of the hyperbolas.

Proposition 3.2. Suppose $G<0, \gamma<0$. Then one intersection is in quadrant $I I$ and one intersection is in quadrant $I V$. Thus, one wave moves to the right and decays while a second wave moves to the left and grows.

Proof: As before, for $G<\frac{1}{4}$, intersection C is necessarily in quadrant II. Regarding the intersection $\mathrm{D}$, first consider the case $\gamma^{2} \xi^{2}<\psi(G)$ (see Fig. 3 Right).

If the hyperbola associated with (3.11a) does not intersect the $\beta$ axis (then necessarily $\gamma^{2} \xi^{2}<2$ ), then the right arm of the hyperbola lies in the right half plane, so the intersection $\mathrm{D}$ of the two hyperbolas occurs in quadrant I or quadrant IV. On the other hand, for $\gamma^{2} \xi^{2} \geq 2$, the hyperbola does intersect the $\beta$ axis, and we wish to establish the same conclusion.

Since $G<0$, the lower intersection of the hyperbola (3.11a) with the $\beta$ axis is $\beta_{a}^{+}$, given by (3.17). Moreover, from (3.18b), we see that the sign of $\beta_{a}^{+}-\beta_{b}$ depends on the sign of

$$
P(G, \gamma \xi)=\left(G-\frac{1}{2}\right)\left[\sqrt{\gamma^{2} \xi^{2}-2}+2 \gamma \xi\left(G-\frac{1}{4}\right)\right] .
$$

But $P(G, \gamma \xi) \neq 0$ since $\gamma^{2} \xi^{2}<\phi(G)$. In the limit $\gamma^{2} \xi^{2} \rightarrow 2^{+}$we find that $P(G, \gamma \xi)>0$. Thus $\beta_{a}^{+}-\beta_{b}>0$ establishing that the intersection $\mathrm{D}$ of the two hyperbolas occurs in quadrant I or quadrant IV. (Note that in Fig. 3 Right, the intersection is shown in quadrant II, which does not occur when $G<0$.)

Next we compare the $\alpha$ values, to show that in fact, the intersection point $D$ lies in quadrant IV.

From (3.18a), we observe that the sign of $\alpha_{a}^{+}-\alpha_{b}$ depends on the sign of

$$
\tilde{Q}(G)=2 G-\frac{1}{2}+\sqrt{\left(G-\frac{1}{2}\right)^{2}+2 G^{2}}
$$

and following the procedure used with (3.21) we find $\tilde{Q}<0$ when $G=-1$ which means $\alpha_{a}-\alpha_{b}<0$. Consequently, the intersection point cannot lie in quadrant I, and must lie in quadrant IV. In the companion case, as for the earlier proposition, the comparisons work the same way, even though the hyperbolas are oriented differently (see Fig. 3 Left).

3.3. Numerical results. Numerical simulations of the system of equations (1.3) help confirm the predictions of the analysis of the dispersion relation. In the simulations, we introduce a small smooth localized perturbation in $h$ into the triple-step traveling wave, away from the jumps, and integrate the equations using a finite difference method that couples an explicit upwind scheme for the convective terms and an implicit scheme for the time-step and the parabolic terms [10]. We use the standard notation for spatial averages of $u_{j}^{n}=u\left(x_{j}, t_{n}\right)$,

$$
\bar{u}_{j+1 / 2}^{n} \equiv \frac{u_{j+1}^{n}+u_{j}^{n}}{2} .
$$


The nonlinear system

$$
\begin{aligned}
& (3.25) h_{j}^{n+1}-h_{j}^{n}-\Delta t\left[\frac{\left(\bar{h}_{j+1 / 2}^{n+1}\right)^{2}\left(\frac{\Gamma_{j+1}^{n+1}-\Gamma_{j}^{n+1}}{\Delta x}\right)-\left(\bar{h}_{j-1 / 2}^{n+1}\right)^{2}\left(\frac{\Gamma_{j}^{n+1}-\Gamma_{j-1}^{n+1}}{\Delta x}\right)}{2 \Delta x}\right] \\
& +\Delta t\left(\frac{\left(h_{j}^{n}\right)^{3}-\left(h_{j-1}^{n}\right)^{3}}{3 \Delta x}\right)-\epsilon \Delta t \frac{\left(h_{j+1}^{n+1}-2 h_{j}^{n+1}+h_{j-1}^{n+1}\right)}{\Delta x^{2}}=0 \\
& \left(3_{j}^{n+26}\right)-\Gamma_{j}^{n}-\Delta t\left[\frac{\bar{h}_{j+1 / 2}^{n+1} \bar{\Gamma}_{j+1 / 2}^{n+1}\left(\frac{\Gamma_{j+1}^{n+1}-\Gamma_{j}^{n+1}}{\Delta x}\right)-\bar{h}_{j-1 / 2}^{n+1} \bar{\Gamma}_{j-1 / 2}^{n+1}\left(\frac{\Gamma_{j}^{n+1}-\Gamma_{j-1}^{n+1}}{\Delta x}\right)}{\Delta x}\right] \\
& +\Delta t\left[\frac{\left(h_{j}^{n}\right)^{2} \Gamma_{j}^{n}-\left(h_{j-1}^{n}\right)^{2} \Gamma_{j-1}^{n}}{2 \Delta x}\right]=0 .
\end{aligned}
$$

is solved using Newton's method. An artificial diffusion term is included at the end of the $h$ equation in order to suppress spatial oscillations near jumps in $h$. In our simulations, we used $\epsilon=0.001$.
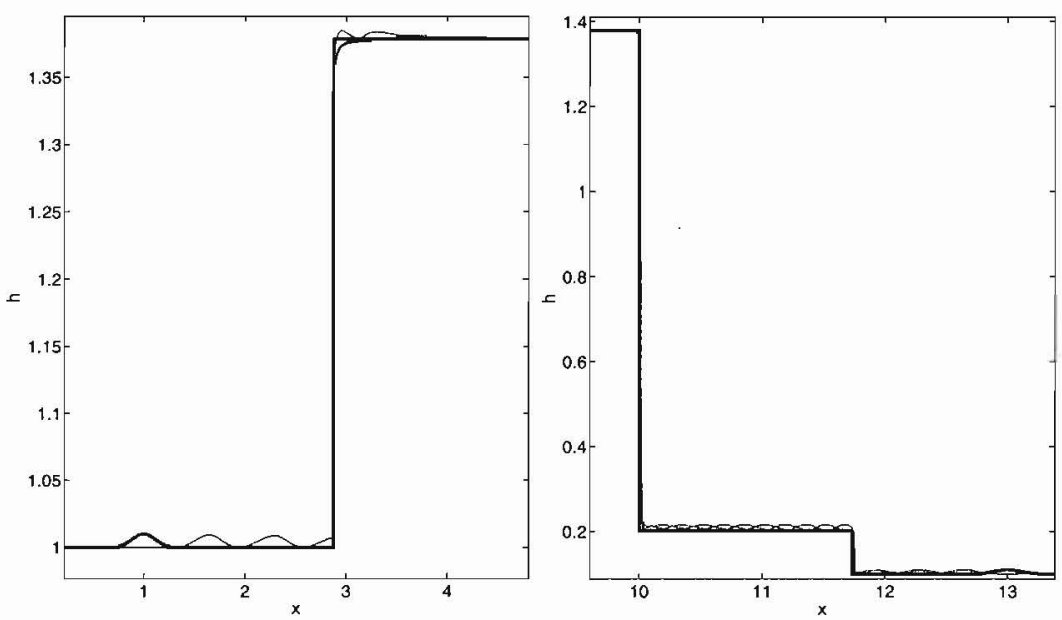

Figure 4. Left: Perturbation placed on $h_{L}$. Right: Perturbation placed on $h_{R}$. Thicker line is initial condition.

When introduced ahead of or behind the traveling wave, the perturbation propagates towards the middle of the wave from either side, as predicted by the equations linearized around $h=$ constant, $\Gamma=0$ (see (2.8) above). The perturbation then encounters the outer discontinuity in $h$, and passes through with some distortion, as seen in Figure 4. Subsequently, we are in the domain of the analysis of the previous subsection, except of course the numerical simulations are not tracking a perturbation with a single wave number, but rather a composition of all wave numbers, with the emphasis being on low wavenumbers - the perturbation is a long wave. When the perturbation is introduced on the levels $h=h_{1}, h=h_{2}$, the evolution is exhibited in Figure 5. On the higher level $\left(h=h_{1}\right)$, the perturbation dies out 

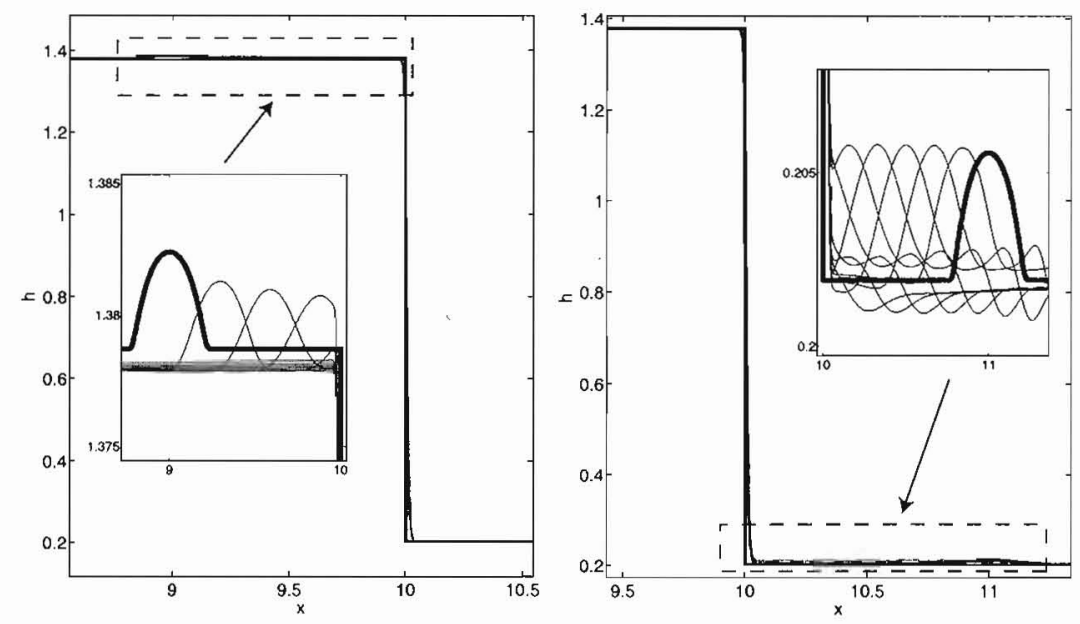

FIGURE 5. Left: Perturbation is placed on $h_{1}$. Right: Perturbation is placed on $h_{2}$.

rapidly, whereas on the lower level $\left(h=h_{2}\right)$, the perturbation propagates towards the big central jump in $h$ and is absorbed by it. On this level, there is presumably a small decaying wave moving right as well, but we have not observed it, possibly because it is a small effect at long wavelengths.

\section{Discussion}

Since the triple-step wave is readily captured in numerical simulations $[\mathbf{1 2}, \mathbf{1 3}]$, it should be expected to be stable to one-dimensional perturbations. If it were a shock wave solution of a hyperbolic conservation law (thus with a single step), then stability would be related to short-time preservation of the shock structure under perturbation of initial conditions; such stability is typically associated with the Lax entropy condition, which guarantees that perturbations in the characteristic family of the shock are transported into the shock, where they are absorbed, while other components of the perturbation are transported through the shock and away. However, the traveling wave under consideration here is not a hyperbolic wave, but a traveling wave solution of a system which in essence (in the absence of higher order derivatives) is a scalar conservation law coupled to a degenerate diffusion equation akin to the porous medium equation. At this quasi-hyperbolic level, the new traveling wave behaves like an overcompressive shock. In Section 2 we observe that in the linearized equations, perturbations ahead and behind the wave converge on the wave, as a pair of traveling waves, much as perturbations of a shock travel along characteristics. However, if a perturbation is placed within the traveling wave, then it is not so clear how the solution evolves. In Section 3 we analyze the linearized system at the level of the dispersion relation, which contains information about the direction and growth or decay of perturbations of a specified frequency. In summary, we find that the only perturbations that can grow propagate towards the middle layer. Numerical simulations show that such perturbations are absorbed by the middle layer.

The analysis of Section 3 is of course not definitive. It would be more satisfactory to have analysis of spectral stability through an understanding of the spectrum 
of the equations linearized around a smooth traveling wave. It will be interesting to see how far an analysis of the Evans function can be pursued; it will be somewhat simpler in the case of negligible surface tension $(\kappa=0)$ since the ODEs are then lower order (see [4]). It would also be interesting to analyze features of instability to transverse perturbations, as was done numerically for a similar problem by Edmonstone, Craster and Matar [6]. As in the surfactant-free case [2], it is to be expected that all of these traveling waves with small amounts of surfactant are unstable unless $\beta>0$ is sufficiently large compared to $\kappa$. However, because of the triple-step structure of the wave, it is not immediately clear what will control multidimensional stability for larger amounts of surfactant.

\section{References}

[1] R. Abeyaratne and J. K. Knowles, Kinetic relations and the propagation of phase boundaries in solids. Arch. Rational Mech. Anal. 114 (1991), 119-154.

[2] A. L. Bertozzi and M. P. Brenner, Linear stability and transient growth in driven contact lines. Physics of Fluids 9 (1997), 530-539.

[3] A. L. Bertozzi, A. Münch and M. Shearer, Undercompressive Shocks in Thin Film Flows, Physica D, 134 (1999), 431-464.

[4] A. L. Bertozzi, A. Münch, M. Shearer and K. Zumbrun, Stability of compressive and undercompressive thin film travelling waves. European J. Appl. Math. 12 (2001), 253-291.

[5] P. G. de Gennes, Wetting: statics and dynamics. Reviews of Modern Physics 57 (1985), 827-863.

[6] B. Edmonstone, R.V. Craster and O. Matar, Surfactant-induced fingering phenomena in thin film flow down an inclined plane. Physica D 209 (2005), 62-79.

[7] D. P. Gaver and J. B. Grotberg, Droplet spreading on a thin viscous film. J. of Fluid Mech. 235 (1992), 399-414.

[8] P.G. LeFloch, Hyperbolic systems of conservation laws: The theory of classical and nonclassical shock waves, Lectures in Mathematics, Birkhäuser, 2002.

[9] P.G. LeFloch and M. Shearer, Nonclassical Riemann solvers with nucleation. Proc. Roy. Soc. Edinburgh., 134A (2004), 961-984.

[10] R. Leveque, Finite Difference Methods for Ordinary and Partial Differential Equations. SIAM, (2007).

[11] R. Levy and M. Shearer, Kinetics and nucleation for driven thin film flow. Physica D, 209 (2005), 145-163.

[12] R. Levy and M. Shearer, The motion of a thin liquid film driven by surfactant and gravity. SIAM J. Appl. Math., 66 (2006), 1588-1609.

[13] R. Levy, M. Shearer, and T. Witelski, Gravity-driven thin liquid films with insoluble surfactant: smooth traveling waves. European J. Appl. Math. 18 (2008), 679-708.

[14] D. Serre, Systems of Conservation Laws 1: Hyperbolicity, Entropies, Shock Waves. Cambridge University Press, 1999.

[15] S. Schecter and V. Manukian, Traveling waves for a thin liquid film with surfactant on an inclined plane. Preprint, 2008.

[16] L. W. Schwartz and R. V. Roy, Theoretical and numerical results for spin coating of viscous liquids. Physics of Fluids 16 (2004), 569-584.

[17] T.M. Segin, B.S. Tilley, and L. Kondic, On undercompressive shocks in constrained two-layer flows. Physica D 209 (2005), 245-259.

[18] H. A. Stone, A simple derivation of the time-dependent convective-diffusion equation for surfactant transport along a deforming interface, Physics of Fluids A 2 (1990), 111-112. 
868 ELLEN PETERSON, MICHAEL SHEARER, THOMAS WITELSKI, AND RACHEL LEVY

Dept. of Mathematics and Center for Research in Scientific Computation, N.C. State UnIVersity, Raleigh, NC 27695.

Dept. of Mathematics and Center for Research in Scientific Computation, N.C. State University, Raleigh, NC 27695.

Dept. of Mathematics and Center for Nonlinear and Complex Systems, Duke UniVERSITY, DURHAM NC 27708-0320.

Dept. of Mathematics, Harvey-Mudd College, Claremont, California. 